\title{
Intensity-modulated radiation therapy achieves better local control compared to three-dimensional conformal radiation therapy for T4-stage nasopharyngeal carcinoma
}

\author{
Jenny Ling-Yu Chen ${ }^{1,2}$, Yu-Sen Huang ${ }^{3,4}$, Sung-Hsin Kuo ${ }^{1,5}$, Ruey-Long Hong ${ }^{1}$, \\ Jenq-Yuh Ko ${ }^{6}$, Pei-Jen Lou ${ }^{6}$ and Chun-Wei Wang ${ }^{1}$ \\ ${ }^{1}$ Department of Oncology, National Taiwan University Hospital and National Taiwan University College of Medicine, Taipei, \\ Taiwan \\ 2 Department of Radiation Oncology, National Taiwan University Hospital Hsin-Chu Branch, Hsin-Chu, Taiwan \\ 3 Department of Medical Imaging, National Taiwan University Hospital and National Taiwan University College of Medicine, \\ Taipei, Taiwan \\ ${ }^{4}$ Department of Medical Imaging, National Taiwan University Hospital Yun-Lin Branch, Yun-Lin, Taiwan \\ ${ }^{5}$ Graduate Institute of Oncology, National Taiwan University, Taipei, Taiwan \\ ${ }^{6}$ Department of Otolaryngology, National Taiwan University Hospital and National Taiwan University College of Medicine, \\ Taipei, Taiwan \\ Correspondence to: Chun-Wei Wang, email: cwwang@ntuh.gov.tw \\ Keywords: intensity-modulated radiation therapy; conformal radiation therapy; nasopharyngeal carcinoma; survival outcomes; \\ late toxicities \\ Received: May 23, $2016 \quad$ Accepted: October 12, $2016 \quad$ Published: October 18, 2016
}

\section{ABSTRACT}

Purpose: To examine the survival outcomes and late toxicity profiles of threedimensional conformal radiation therapy (3DCRT) vs. intensity-modulated radiation therapy (IMRT) for patients with nasopharyngeal carcinoma (NPC).

Methods: Three hundred and seventy-four patients with newly diagnosed, nonmetastatic, NPC who were curatively treated with 3DCRT between 2004 and 2006 and 481 patients treated with IMRT between 2007 and 2009 were analyzed. Patients were categorized as having advanced-stage disease (stage III, IVA, and IVB disease; $n=709$ ) or early-stage disease (stage I and II; $n=146$ ). The median follow-up time was $\mathbf{9 0 . 3}$ months for patients treated with 3DCRT and 86.3 months for patients treated with IMRT.

Results: For early-stage patients, the outcomes of IMRT vs. 3DCRT were similar considering locoregional control (LRC), distant metastasis-free survival (DMFS), and overall survival (OS). For advanced-stage patients, IMRT was associated with better LRC compared with 3DCRT (5-year LRC rate: $85.6 \%$ vs. $76.6 \%$, respectively; $p=0.035$ ) and OS (5-year OS rate: $82.3 \%$ vs. $71.8 \%$, respectively; $p=0.002$ ),

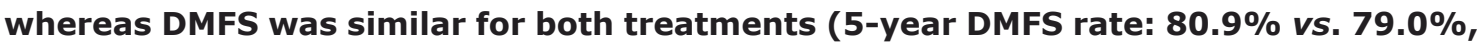
respectively; $p=0.324$ ). Furthermore, the IMRT technique was more beneficial for patients with T4 disease. Late toxicities occurred more frequently in patients treated with 3DCRT than in those treated with IMRT (grade $\geq 3$ neck fibrosis: $6.7 \%$ vs. $3.7 \%$, respectively, $p=0.036$; radiographic temporal lobe necrosis: $10.2 \%$ vs. $4.4 \%$, respectively, $p<0.001$ ).

Conclusions: Compared with 3DCRT, IMRT offered better LRC in patients with advanced-stage non-metastatic NPC, which corresponded with better OS. 


\section{INTRODUCTION}

Nasopharyngeal carcinoma (NPC) is a highly radiosensitive tumor, and definitive radiation therapy (RT) is the standard treatment for it [1,2]. Modern RT techniques have emerged with the development of conformal RT (CRT), such as three-dimensional CRT (3DCRT) or intensity-modulated RT (IMRT). CRT allows better delineation of the tumor target and organs at risk with clearer radiologic visualization of their spatial relations, thus providing a potential therapeutic benefit of dose escalation to tumor tissues, with reduced toxicity to normal tissues.

IMRT achieves better dose differentiation between tumorous and normal tissues compared with 3DCRT and facilitates simultaneous delivery of different fractional doses to different targets [3,4]. In addition, IMRT has the advantage of better tumor coverage because it allows for dose escalation, while reducing exposure to the parotid gland, temporomandibular joints, and brainstem/temporal lobe. Thus, IMRT is superior to 3DCRT in that it increases the biologic effect on the tumor owing to physical dose escalation, while avoiding toxicity to critical tissues $[5,6]$.

The technical and dosimetric superiority of RT techniques could translate into clinical benefits, such as reduced normal tissue toxicity, improved quality of life, and increased locoregional control (LRC) and survival outcomes [7-11]. Despite the potential advantages of IMRT for NPC, the exact survival benefit and late toxicity reduction after treatment with IMRT compared with 3DCRT are not known; in addition, there is no evidence about which patient population would most benefit from IMRT treatment. The study aimed to compare the survival outcomes and late toxicity profiles after treatment with 3DCRT and IMRT for patients with NPC.

\section{RESULTS}

\section{Patient characteristics}

A total of 855 NPC patients who were treated with curative intent with 3DCRT $(n=374)$ between 2004 and 2006 or with IMRT $(n=481)$ between 2007 and 2009 were analyzed. Patient and treatment characteristics are listed in Table 1 . The median patient age was 49 years (range, 10-93 years). Four patients ( 2 in the 3DCRT group and 2 in the IMRT group) developed life-threatening sepsis and expired before completing radiotherapy. These four patients had a follow-up time of 0 months. The median follow-up time was 90.3 months (range, 0-111.2 months) for patients treated with 3DCRT and 86.3 months (range, 0-111.4 months) for patients treated with IMRT. As shown in Table 1, compared with the patients in the 3DCRT group, patients in the IMRT group were diagnosed at earlier T and $\mathrm{N}$ stages $(p<0.001$ and $p=$ 0.017 , respectively). Advanced-stage disease (stage III, IVA, and IVB) was more commonly observed in the 3DCRT group (92.0\% vs. $75.9 \%$ in the IMRT group, $p$ $<0.001)$. These chronologic epidemiologic data suggest the implementation of nasopharyngeal screening during clinical practice. Of the 349 patients in the 3DCRT group and 452 patients in the IMRT group who had concurrent systemic therapy, 32 patients and 29 patients, respectively, received $100 \mathrm{mg} / \mathrm{m}^{2}$ cisplatin every 3 weeks for 2-3 cycles, while the remaining patients received $30 \mathrm{mg} / \mathrm{m}^{2}$ cisplatin every week.

\section{Treatment outcomes}

In the overall population, the median LRC, DMFS, and OS were 79.2 months, 81.7 months, and 83.0 months, respectively. At the time of analysis, 301 patients $(35.2 \%)$ had recurrence at $\geq 1$ sites, and $192(22.5 \%)$ had died of the disease. Univariate and multivariate analyses (Table 2) revealed that old age, advanced stage, and use of the 3DCRT technique were associated with poor outcomes. No significant difference was found for the different concurrent chemotherapy regimens $\left(100 \mathrm{mg} / \mathrm{m}^{2}\right.$ cisplatin every 3 weeks or $30 \mathrm{mg} / \mathrm{m}^{2}$ cisplatin every week) on LRC $(p=0.563)$, DMFS $(p=0.090)$, or OS $(p=0.294)$.

To eliminate the selection bias of more advancedstage patients in the 3DCRT group that would have led to poor survival outcomes, early and advanced-stage patients were separated to compare the two radiation therapy techniques. As shown in Figure 1, for early-stage patients, the outcomes of IMRT and 3DCRT were similar considering the LRC (LRC, 5-year LRC rate: $92.5 \%$ vs. $90.0 \%$, respectively; $p=0.934$ ), DMFS (DMFS, 5 -year DMFS rate: $95.5 \%$ vs. $93.2 \%$, respectively; $p=0.620$ ), and OS (OS, 5-year OS rate: $95.6 \%$ vs. $89.3 \%$, respectively; $p$ $=0.594)$. For advanced-stage patients, IMRT, compared with 3DCRT, was associated with better LRC (5-year LRC rate: $85.6 \% v s .76 .6 \%$, respectively; $p=0.035)$ and OS (5-year OS rate: $82.3 \%$ vs. $71.8 \%$, respectively; $p=$ 0.002 ), whereas DMFS was similar for both the treatments (5-year DMFS rate: $80.9 \%$ vs. $79.0 \%$, respectively; $p=$ $0.324)$. To determine which subgroup of advanced stage cancer would benefit the most from IMRT and 3DCRT, the survival outcomes were compared. As shown in Table 3 , the IMRT technique was more beneficial for patients with T4 disease than for those with T3, N2, or N3 disease. In advanced-stage patients with T4 disease, IMRT was associated with better LRC, compared to 3DCRT (5year LRC rate: $83.4 \%$ vs. $70.6 \%$, respectively; $p=0.048$ ) and OS (5-year OS rate: $77.2 \%$ vs. $65.3 \%$, respectively; $p=0.011$; Figure 2), whereas DMFS was similar for both treatments (5-year DMFS rate: $73.0 \%$ vs. $73.5 \%$, respectively; $p=0.697$ ). 
Table 1: Patient clinical characteristics and treatment parameters

\begin{tabular}{|c|c|c|c|c|c|c|}
\hline & & \multicolumn{2}{|c|}{ 3DCRT $(n=374)$} & \multicolumn{2}{|c|}{ IMRT $(n=481)$} & \multirow[t]{2}{*}{$P$-value } \\
\hline & & $n$ & $\%$ & $n$ & $\%$ & \\
\hline \multicolumn{2}{|c|}{ Age } & & & & & 0.361 \\
\hline & $\leq 60$ years & 302 & 80.7 & 400 & 83.2 & \\
\hline & $>60$ years & 72 & 19.3 & 81 & 16.8 & \\
\hline \multicolumn{2}{|c|}{ Sex } & & & & & 0.149 \\
\hline & Male & 265 & 70.9 & 362 & 75.3 & \\
\hline & Female & 109 & 29.1 & 119 & 24.7 & \\
\hline \multicolumn{2}{|c|}{ T stage } & & & & & $<0.001 *$ \\
\hline & $\mathrm{T} 1$ or $\mathrm{T} 2$ & 76 & 20.3 & 207 & 43.0 & \\
\hline & $\mathrm{T} 3$ or $\mathrm{T} 4$ & 298 & 79.7 & 274 & 57.0 & \\
\hline \multicolumn{2}{|c|}{$\mathrm{N}$ stage } & & & & & $0.017 *$ \\
\hline & $\leq \mathrm{N} 2$ & 277 & 74.1 & 389 & 80.9 & \\
\hline & N3 & 97 & 25.9 & 92 & 19.1 & \\
\hline \multicolumn{2}{|c|}{ Early $v s$. Advanced stage } & & & & & $<0.001 *$ \\
\hline & Early (stage I and II) & 30 & 8.0 & 116 & 24.1 & \\
\hline & $\begin{array}{l}\text { Advanced (stage III, IVA, } \\
\text { and IVB) }\end{array}$ & 344 & 92.0 & 365 & 75.9 & \\
\hline \multicolumn{2}{|c|}{ Duration } & & & & & 0.793 \\
\hline & $\leq 50$ days & 183 & 48.9 & 231 & 48.0 & \\
\hline & $>50$ days & 191 & 51.1 & 250 & 52.0 & \\
\hline \multicolumn{2}{|c|}{ Induction therapy } & & & & & $<0.001 *$ \\
\hline & No & 231 & 61.8 & 384 & 79.8 & \\
\hline & Yes & 143 & 38.2 & 97 & 20.2 & \\
\hline \multicolumn{2}{|c|}{ Concurrent systemic therapy } & & & & & 0.696 \\
\hline & No & 25 & 6.7 & 29 & 6.0 & \\
\hline & Yes & 349 & 93.3 & 452 & 94.0 & \\
\hline
\end{tabular}

Abbreviations: 3DCRT $=$ three-dimensional conformal radiation therapy; IMRT $=$ intensity-modulated radiation therapy. $P$-values obtained by using the Student $t$-test. ${ }^{*} P<0.05$ for comparison between $3 \mathrm{DCRT}$ and IMRT.

Table 2: Univariate and multivariate analyses of potential prognostic factors

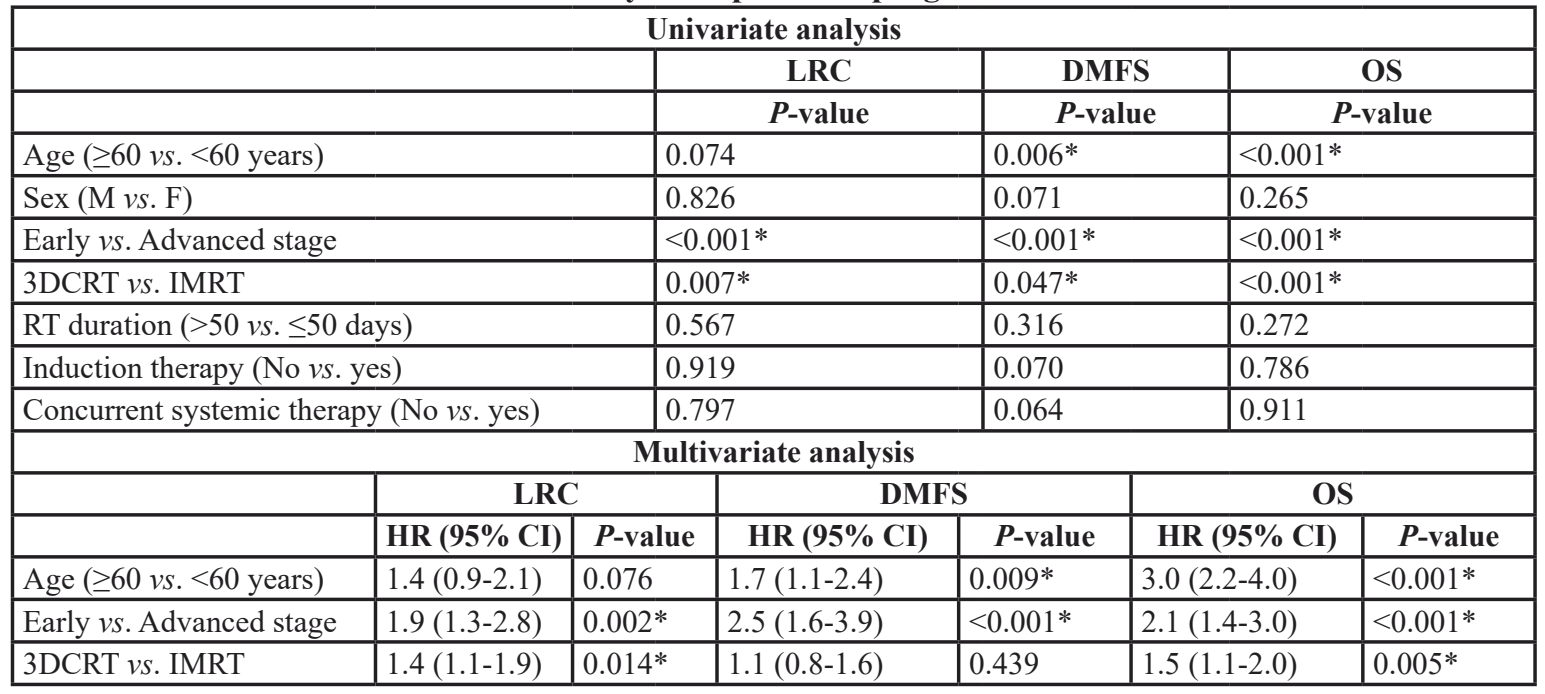

Abbreviations: LRC = locoregional control; DMFS = distant metastasis-free survival; OS = overall survival; HR = hazard ratio; $\mathrm{CI}=$ confidence interval; 3DCRT $=$ three-dimensional conformal radiation therapy; $\mathrm{IMRT}=$ intensity-modulated radiotherapy; $* P<0.05$ with statistical significance.

\section{Radiation therapy late toxicity}

As shown in Table 4 , grade $\geq 3$ toxicities occurred in $27.8 \%$ and $27.5 \%$ of patients treated with IMRT and 3DCRT, respectively $(p=0.787)$. No significant difference was seen considering grade $\geq 3$ ototoxicity, cranial neuropathy, xerostomia, or osteoradionecrosis. In 


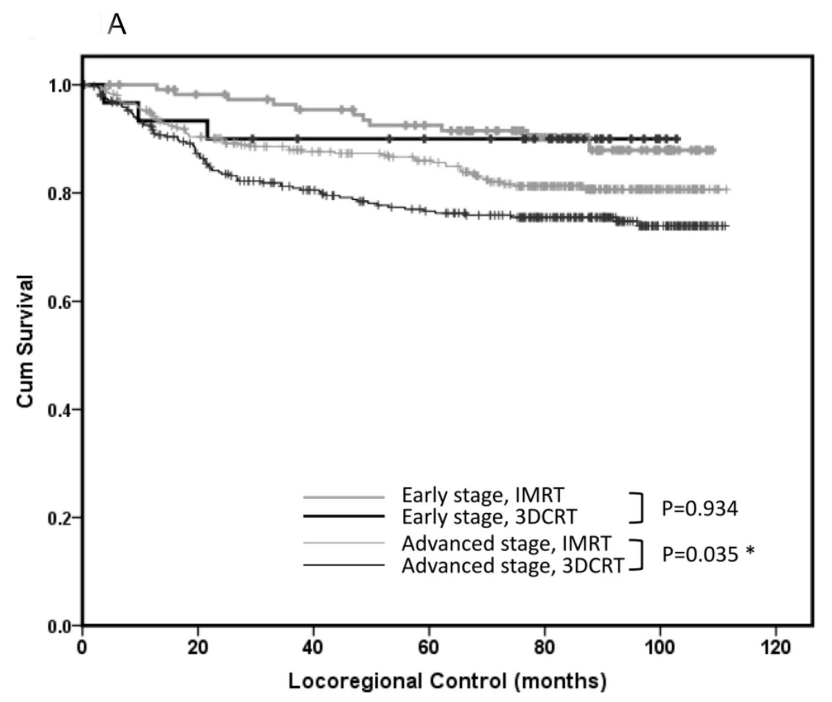

B
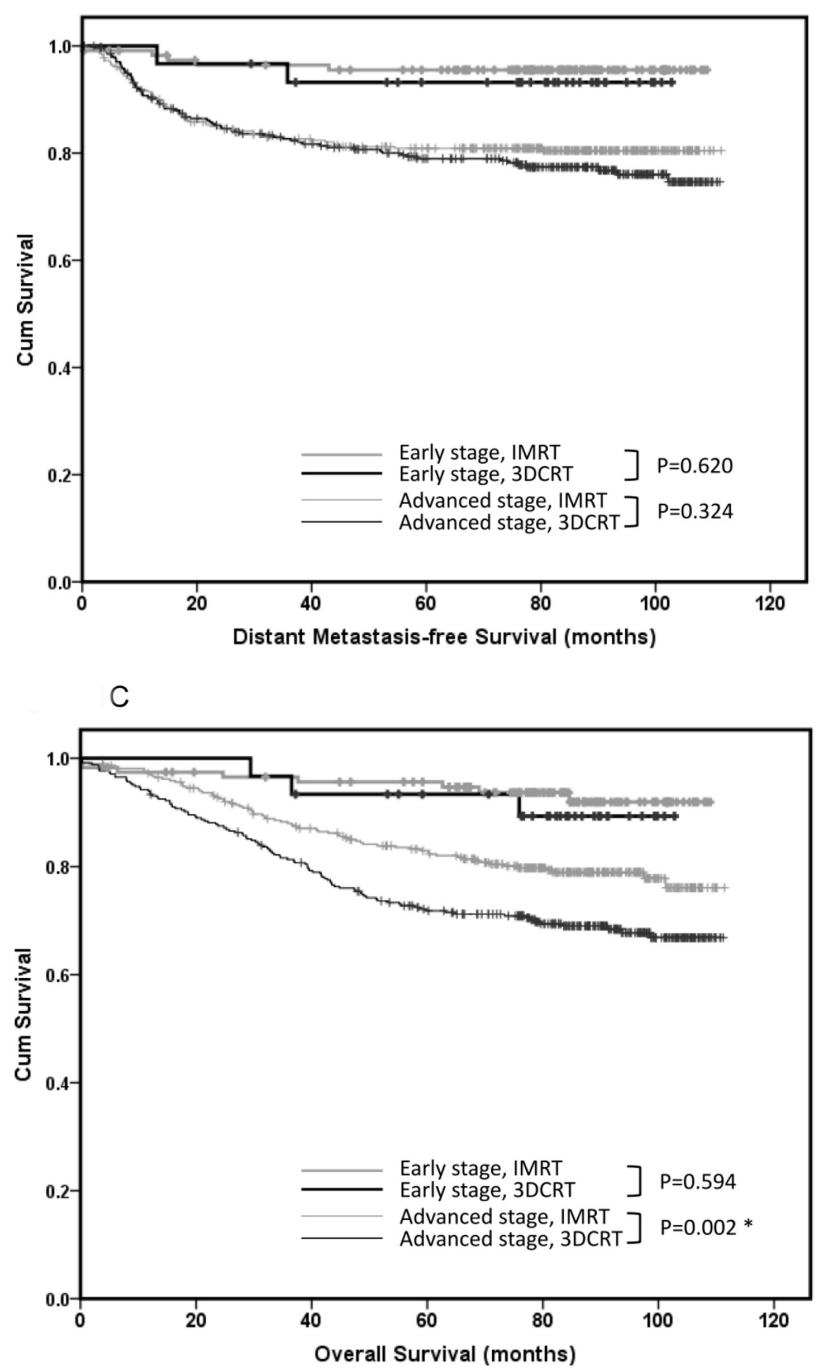

Figure 1: Outcomes of patients with early and advanced-stage nasopharyngeal carcinoma. The outcomes of patients with early and advanced-stage nasopharyngeal carcinoma treated with three-dimensional conformal radiation therapy (3DCRT, $n=374)$ versus those treated with intensity-modulated radiation therapy (IMRT, $n=481$ ) considering A. locoregional control, B. distant metastasis-free survival, and $\mathbf{C}$. overall survival. 
Table 3: Survival outcomes of advanced-stage patients treated with 3DCRT vs. IMRT

\begin{tabular}{|l|l|l|l|l|}
\hline & 5-year survival rate & 3DCRT & IMRT & $P$-value \\
\hline T3 $(n=234)$ & & $n=114$ & $n=120$ & \\
\hline & LRC & $82.2 \%$ & $86.5 \%$ & 0.379 \\
\hline & DMFS & $87.2 \%$ & $85.2 \%$ & 0.952 \\
\hline & OS & $79.7 \%$ & $86.3 \%$ & 0.562 \\
\hline T4 $(n=338)$ & & $n=184$ & $n=154$ & \\
\hline & LRC & $70.6 \%$ & $83.4 \%$ & $0.048 *$ \\
\hline & DMFS & $73.5 \%$ & $73.0 \%$ & 0.697 \\
\hline & OS & $65.3 \%$ & $77.2 \%$ & $0.011^{*}$ \\
\hline & & $n=184$ & $n=178$ & \\
\hline & LRC $(n=362)$ & 81.1 & 88.7 & 0.312 \\
\hline & DMFS & 83.4 & 84.4 & 0.597 \\
\hline & OS & 76.9 & 87.1 & $0.044 *$ \\
\hline N3 $(n=189)$ & & $n=97$ & $n=92$ & \\
\hline & LRC & $71.8 \%$ & $82.6 \%$ & 0.068 \\
\hline & DMFS & $65.4 \%$ & $71.4 \%$ & 0.274 \\
\hline & OS & $60.6 \%$ & $71.7 \%$ & 0.096 \\
\hline
\end{tabular}

Abbreviations: $\mathrm{LRC}=$ locoregional control; DMFS $=$ distant metastasis-free survival; OS $=$ overall survival; $3 \mathrm{DCRT}=$ threedimensional conformal radiation therapy; IMRT = intensity-modulated radiation therapy. $P$-values obtained by using KaplanMeier life table analysis and the log-rank test. ${ }^{*} P<0.05$ for comparison between 3DCRT and IMRT.

Table 4: Radiation therapy late toxicities in patients treated with 3DCRT vs. IMRT

\begin{tabular}{|l|l|l|l|l|l|l|l|}
\hline & \multicolumn{3}{|c|}{ 3DCRT $(\boldsymbol{n}=\mathbf{3 7 4})$} & \multicolumn{3}{c|}{ IMRT $(\boldsymbol{n}=\mathbf{4 8 1})$} & P-value \\
\hline & Grade 3 (\%) & Grade 4 (\%) & Grade 5 (\%) & Grade 3 (\%) & Grade 4 (\%) & Grade 5 (\%) & \\
\hline Otitis/hearing loss & $46(12.3 \%)$ & 0 & 0 & $54(11.2 \%)$ & 0 & 0 & 0.594 \\
\hline Soft tissue fibrosis & $24(6.4 \%)$ & $1(0.3 \%)$ & 0 & $17(3.5 \%)$ & $1(0.2 \%)$ & 0 & $0.036^{*}$ \\
\hline Cranial neuropathy & $27(7.2 \%)$ & $3(0.8 \%)$ & 0 & $38(7.9 \%)$ & 0 & 0 & 0.948 \\
\hline Salivary gland dysfunction & $12(3.2 \%)$ & 0 & 0 & $13(2.7 \%)$ & 0 & 0 & 0.373 \\
\hline Osteonecrosis & $8(2.1 \%)$ & $3(0.8 \%)$ & 0 & $18(3.7 \%)$ & $1(0.2 \%)$ & 0 & 0.426 \\
\hline Epistaxis bleeding & $3(0.8 \%)$ & $7(1.9 \%)$ & $2(0.5 \%)$ & $2(0.4 \%)$ & $8(1.7 \%)$ & $2(0.4 \%)$ & 0.531 \\
\hline Any late toxicities & $86(23.0 \%)$ & $15(4.0 \%)$ & $2(0.5 \%)$ & $120(24.9 \%)$ & $12(2.5 \%)$ & $2(0.4 \%)$ & 0.787 \\
\hline $\begin{array}{l}\text { Radiographic temporal lobe } \\
\text { necrosis }\end{array}$ & $38(10.2 \%)$ & $21(4.4 \%)$ & & & $<0.001 *$ \\
\hline
\end{tabular}

Abbreviations: $3 \mathrm{DCRT}=$ three-dimensional conformal radiation therapy; IMRT $=$ intensity-modulated radiation therapy. $P$-values obtained by using the Pearson chi-square test. ${ }^{*} P<0.05$ for the comparison of $\geq$ Grade 3 toxicities between 3 DCRT and IMRT.

the IMRT and 3DCRT groups, 54 patients $(11.2 \%)$ and $46(12.3 \%)$, respectively, experienced otologic toxicities and required tympanocentesis, grommet insertion, myringotomy, or hearing aids; $38(7.9 \%)$ and $30(8.0 \%)$ developed transient or permanent cranial neuropathy not attributed to locoregional recurrence, and they required hospitalization or more aggressive treatment; $13(2.7 \%)$ and $12(3.2 \%)$ had salivary gland dysfunction without any response to drug stimulation; and 19 (3.9\%) and 11 $(2.9 \%)$ patients experienced osteonecrosis necessitating medication, hyperbaric oxygen, or operative intervention. In total, 18 patients $(3.7 \%)$ in the IMRT group versus $25(6.7 \%)$ in the 3DCRT group experienced soft tissue toxicities with severe induration and loss of subcutaneous tissue, thereby requiring aggressive rehabilitation ( $p=$ 0.036). Radiographic temporal lobe necrosis occurred more frequently in patients treated with 3DCRT than in those treated with IMRT $(10.2 \%$ vs. $4.4 \%$, respectively; $p<0.001)$.

\section{DISCUSSION}

The current study - a large series that focused on patients with non-metastatic NPC treated using 3DCRT or IMRT - was successful in determining the efficacy of modern radiation techniques considering the survival outcomes and late toxicities in patients treated with NPC. Although several studies have demonstrated that IMRT has better dose distributions compared with 3DCRT, the clinical outcome of increasing LRC is still under investigation [7-12]. A retrospective study had shown the benefit of IMRT over two-dimensional radiation therapy (2DRT) considering LRC in early T-stage patients 


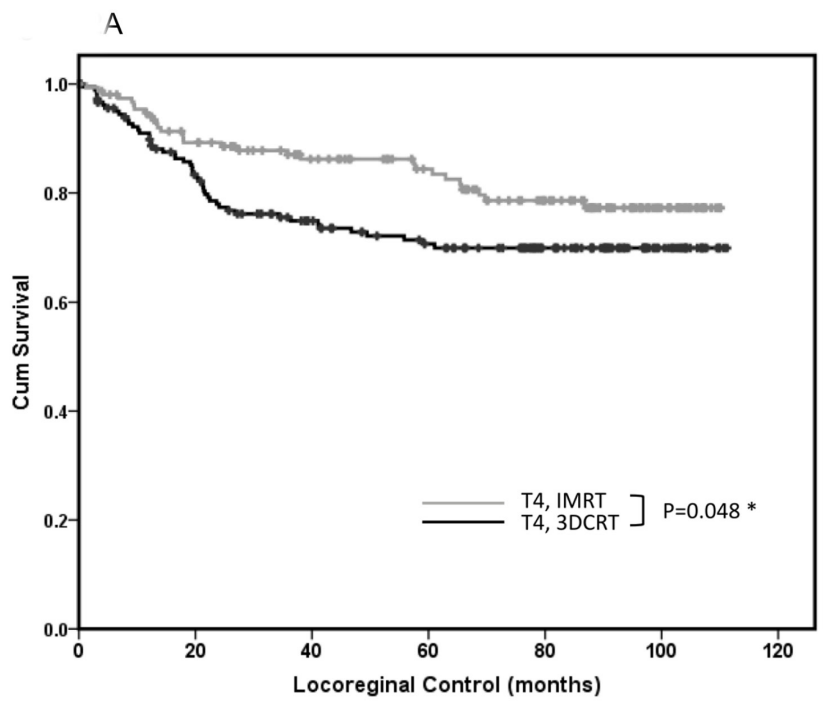

B
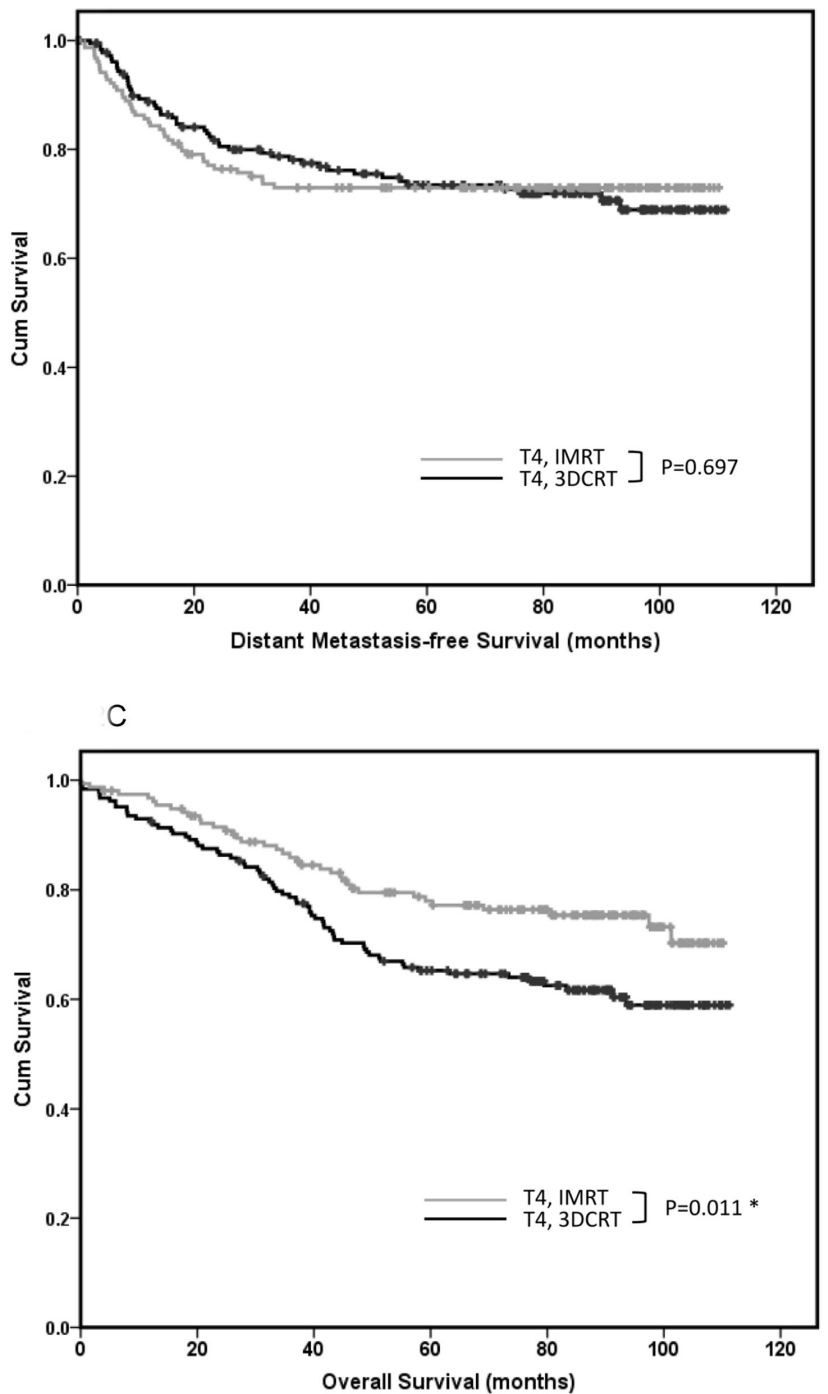

Figure 2: Outcomes of patients with T4 nasopharyngeal carcinoma. The outcomes of patients with T4 nasopharyngeal carcinoma treated with three-dimensional conformal radiation therapy (3DCRT, $n=184$ ) versus those treated with intensity-modulated radiation therapy (IMRT, $n=154$ ) considering A. locoregional control, B. distant metastasis-free survival, and C. overall survival. 


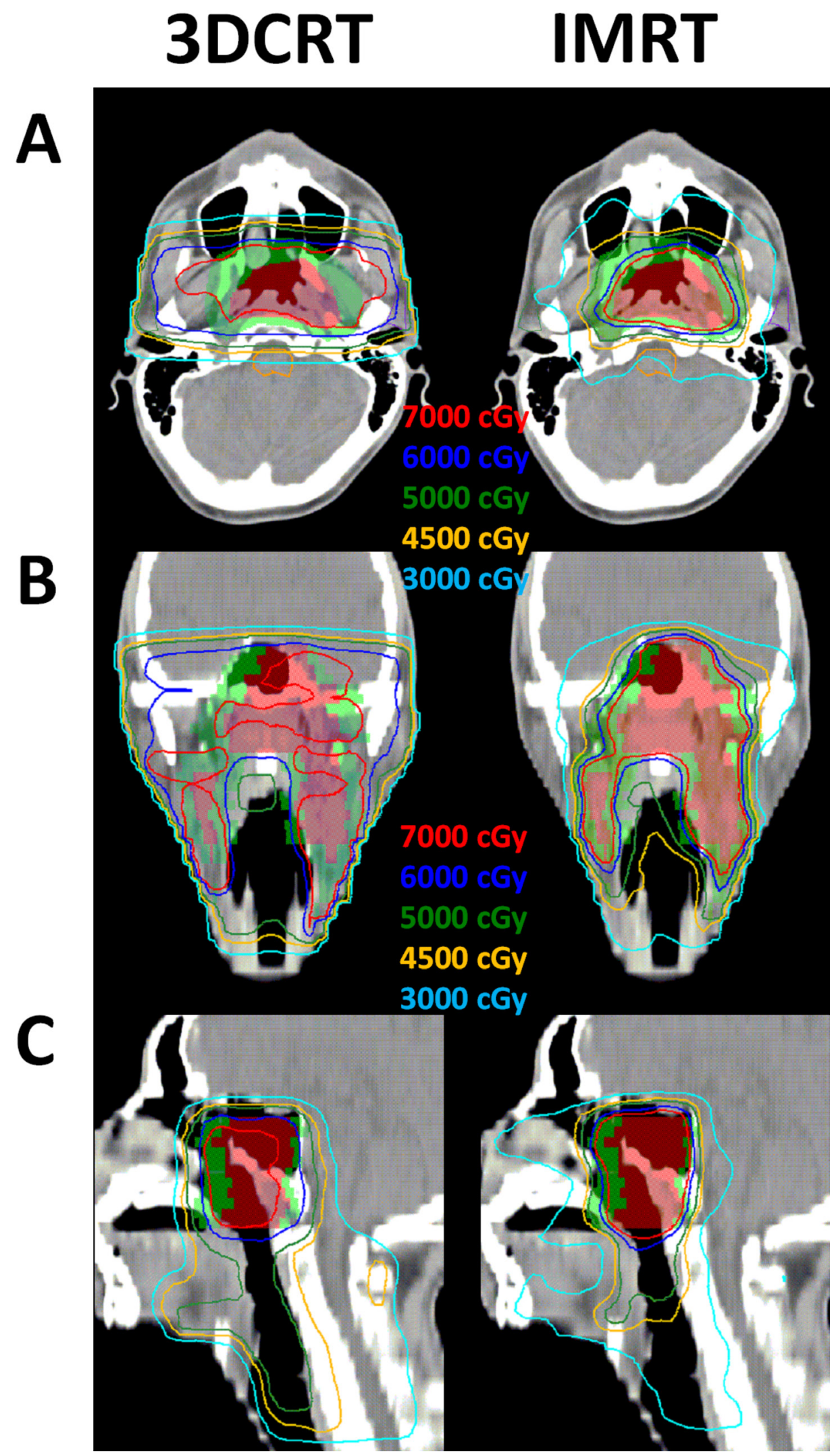

Figure 3: Isodose distributions in one NPC patient. The isodose distributions for one NPC patient (cT3N2M0) planned by 3DCRT (left) and IMRT (right) displayed on the A. axial, B. coronal, and C. sagittal planes. Color-wash areas: CTV-70: red; CTV-50: green. The red, blue, green, orange, and indigo lines were isodose curves of $70 \mathrm{~Gy}, 60 \mathrm{~Gy}, 50 \mathrm{~Gy}, 45 \mathrm{~Gy}$, and $30 \mathrm{~Gy}$. 
[9]; however, other studies comparing IMRT and 2DRT or 3DCRT had shown the benefit of IMRT for LRC in advanced patients $[10,13]$, which is in line with our findings. IMRT planning has the advantage of achieving steeper dose gradients between tumorous and normal tissues, and thus, IMRT results in good LRC in patients with T4 NPC $[3,4]$.

In our series, IMRT reduced the frequency of late toxicities of RT, including severe neck fibrosis and radiographic temporal lobe necrosis, which is in line with the findings of several retrospective studies that revealed a better quality of life after IMRT compared with that obtained after 3DCRT [7, 8, 11]. Other studies comparing IMRT and 2DRT have also shown decreased temporal lobe injury, cranial neuropathy, trismus, neck fibrosis, xerostomia, or hearing loss in the IMRT group [9-11, 14].

Our study has several limitations, including the retrospective design. The patient population included was heterogeneous considering the stage, induction chemotherapy, concurrent chemotherapy regimens, and multimodality treatments. Efforts were made to obtain equivalent follow-up times in the different radiotherapy groups, in order to eliminate bias related to timing. The plasma Epstein-Barr virus (EBV) DNA measurement has been incorporated into standard medical practice in several medical centers to help diagnose, monitor, and predict NPC patients' survival outcomes [15-17]. Our institution has obtained EBV DNA measurements since 2014; therefore, no comprehensive data were available regarding EBV DNA measurements for the patient cohort in this study, which retrospectively included patients from 2004 to 2009 . We plan to incorporate these data in future clinical investigations, gaining valuable insights.

IMRT is regarded as the standard care for NPC patients in most developed countries. Owing to the uneven distribution of economic medical resources, a small number of patients are still being treated with 3DCRT technique in the modern era. Our results showed that 3DCRT achieved equivalent treatment outcomes for earlystage patients, compared to IMRT; however, 3DCRT might not be as effective for improving advanced-stage patients LRC, compared to IMRT, indicating that 3DCRT might be sufficient for early-stage patients, while IMRT is ideal for T4 or advanced-stage patients. Furthermore, when economical resources are limited, choosing 3DCRT for early-stage patients and IMRT for advanced-stage patients might be the optimal approach. More evidence is needed to enhance the continuous and comprehensive evolution of radiation therapy, bringing improved approaches and outcomes. We believe that our study may provide a modest contribution to the basis of modern radiation treatment, with emphasis on refinement and precision.

In conclusion, the results of our study suggest that IMRT offered good LRC in patients with advanced-stage non-metastatic NPC, especially in T4 patients, which corresponded with better OS.

\section{MATERIALS AND METHODS}

\section{Patients}

This study was approved by the Institutional Review Board of our institute (201310016RINA). As per institutional policy, patients with NPC were treated with IMRT instead of 3DCRT since January 2007. Accordingly, 374 patients with newly diagnosed, non-metastatic NPC who were curatively treated with 3DCRT between 2004 and 2006 and 481 patients treated with IMRT between 2007 and 2009 were analyzed. According to the American Joint Commission on Cancer classification system, seventh edition [18], patients were categorized as having advanced-stage disease (stage III, IVA, or IVB disease; $n=709$ ) or early-stage disease (stage I and II; $n=146$ ). Staging evaluations included magnetic resonance imaging (MRI) of the nasopharynx and neck, chest radiography, liver ultrasonography, and bone scintigraphy, as well as positron emission tomography/computed tomography (CT), if clinically required.

\section{Radiation therapy}

The RT treatment technique used to treat the patients evaluated in this study has been previously described [1, $4,5]$. Briefly, a thermoplastic mask was used to ensure immobilization of the head to the shoulder. CT was performed with a 3-5-mm slice thickness of the head and neck region. For patients treated with 3DCRT, the initial treated target volume was the gross target volume with a 2-cm margin in all directions, and shrinkage to avoid excessive irradiation to the pons and spinal cord after 46 Gy. All patients were treated with bilateral opposing portals to cover the primary tumor and the neck; the fraction size was $2 \mathrm{~Gy}$. After $36 \mathrm{~Gy}$, the primary tumor and the neck were treated with a split-field technique. Regarding shrinkage, the primary tumor was irradiated with bilateral opposing fields, using $2.5 \mathrm{~Gy}$ as the fraction size; an additional 10 Gy was administered. An additional 24 Gy in 10 fractions was delivered to the nasopharynx via bilateral anterior oblique infraorbital portals. The accumulated radiation dose to the nasopharynx was 70 Gy in 32 fractions. For patients with N0 to N3a disease, the neck was further treated by using anterior-posterior opposing portals to administer 36 Gy in 18 fractions, with the spinal cord shielded. The accumulated dose was 50 Gy in 25 fractions to the uninvolved neck and $60 \mathrm{~Gy}$ in 30 fractions to the involved regions. After $60 \mathrm{~Gy}$, an additional 5 Gy in 2 fractions was administered to the residual neck masses. 3DCRT was delivered using the 6-MV linear accelerator Siemens Mevatron (Siemens AG, Berlin, Germany).

For patients treated with IMRT, the target areas 
received the following 3 dose levels with simultaneous integrated boosts, in accordance with the RTOG 0225 protocol [2]: clinical target volume (CTV)-70 (70 Gy for the gross nasopharyngeal tumor and lymphadenopathy); CTV-63/60 (60-63 Gy for subclinical disease and highrisk lymphatic regions, including the entire nasopharynx, retropharyngeal nodal regions, skull base, clivus, pterygoid fossae, parapharyngeal space, sphenoid sinus, and posterior nasal cavity/maxillary sinuses that include the pterygopalatine fossa and upper middle neck regions; a concentric volume that completely encompassed the entire CTV-70 in all directions); and CTV-56/54 (54-56 Gy to the low-risk regions, including the low/middle neck and supraclavicular fossa regions). The planning target volumes were evenly expanded using a 4-mm margin. Two dose fractionations (33 or 35 fractions) were used, at the discretion of the treating physician. The plans were optimized using an inverse planning algorithm (Direct Machine Parameter Optimization) and heterogeneity corrections. The isodose distributions for NPC patients planned by 3DCRT and IMRT are demonstrated in Figure 3. IMRT was delivered using an Elekta Synergy accelerator (Elekta, Stockholm, Sweden) with a stepand-shoot technique. The treatment position was verified weekly by using cone-beam CT X-ray volume imaging.

\section{Chemotherapy}

Concurrent chemoradiotherapy regimens included $30 \mathrm{mg} / \mathrm{m}^{2}$ cisplatin weekly during radiotherapy or $100 \mathrm{mg} / \mathrm{m}^{2}$ cisplatin every 3 weeks for 2-3 cycles, at the discretion of the chemo-oncologist. Neoadjuvant chemotherapy was administered mainly for patients with obvious intracranial invasion, supraclavicular or bilateral neck lymph node metastasis, or large neck nodes $(>6$ $\mathrm{cm})$ [1], at the discretion of the chemo-oncologist. The induction chemotherapy regimens were $\operatorname{MEPFL}\left(8 \mathrm{mg} / \mathrm{m}^{2}\right.$ mitomycin, $60 \mathrm{mg} / \mathrm{m}^{2}$ epirubicin, and $60 \mathrm{mg} / \mathrm{m}^{2}$ cisplatin on day 1 , and $450 \mathrm{mg} / \mathrm{m}^{2}$ fluorouracil and $30 \mathrm{mg} / \mathrm{m}^{2}$ leucovorin on day 8$)$ or PF $\left(100 \mathrm{mg} / \mathrm{m}^{2}\right.$ cisplatin on day 1 and $1000 \mathrm{mg} / \mathrm{m}^{2}$ fluorouracil on days 1-3) every 3 weeks for 2 or 3 cycles. None of the patients received adjuvant chemotherapy.

\section{Follow-up assessment}

Acute toxicities were rated according to the Radiation Therapy Oncology Group (RTOG) criteria [19]. All patients were followed-up every 2-3 months for the first 2 years, every 4 months for the third year, and every 6 months after the third year until recurrence or death. After completion of treatment, the treatment response after radiotherapy was assessed every 3 months by using endoscopy and head and neck MRI as well as a biopsy of the nasopharynx if suspicions of recurrence were identified. Chest radiographs were taken every 6 months, whereas CT, MRI, bone scanning, or other investigations were performed when clinical suspicions of recurrence were identified. Late toxicities were assessed according to the late morbidity scoring criteria of RTOG.

\section{Statistical analysis}

Statistical analysis was performed using the Statistical Package for Social Sciences for Windows, version 17.0 (SPSS, Chicago, IL). Survival data were confirmed with the Cancer Registry Medical Information Management Office in our hospital. All events were calculated from the date of treatment completion. To obtain equivalent follow-up times for both groups, an analysis was conducted using the follow-up data available on June 20, 2015 for 3DCRT patients and on July 30, 2016 for IMRT patients. Actuarial estimates of LRC, distant metastasis-free survival (DMFS), and overall survival (OS) were calculated using the Kaplan-Meier method and compared using the log rank test. The log-rank test was used to determine the prognostic factors affecting survival. All prognostic variables found to be significant in univariate analysis were included in multivariate analysis using the Cox proportional hazards regression model. A $p$-value of $\leq 0.05$ was considered statistically significant.

\section{ACKNOWLEDGMENTS}

We thank Dr. Ya-Fang Chen, Dr. Chiao-Ling Tsai, and Dr. Wan-Yu Chen for their guidance in conducting this work. The work was presented in part at the 2014 Multidisciplinary Head and Neck Cancer Symposium (Phoenix, USA, February 20-22, 2014).

\section{CONFLICTS OF INTEREST}

The authors indicate no potential conflicts of interest.

\section{GRANT SUPPORT}

This study was supported by the following research grants: the National Taiwan University Hospital (grant NTUH. 104-S2763) and Ministry of Science and Technology (MST, Taiwan, under contract of MST 1012314-B-002-152 and 102-2314-B-002 -151 -MY3).

\section{REFERENCES}

1. Hong RL, Ting LL, Ko JY, Hsu MM, Sheen TS, Lou PJ, Wang CC, Chung NN and Lui LT. Induction chemotherapy with mitomycin, epirubicin, cisplatin, fluorouracil, and 
leucovorin followed by radiotherapy in the treatment of locoregionally advanced nasopharyngeal carcinoma. J Clin Oncol. 2001; 19:4305-4313.

2. Lee N, Harris J, Garden AS, Straube W, Glisson B, Xia P, Bosch W, Morrison WH, Quivey J, Thorstad W, Jones C and Ang KK. Intensity-modulated radiation therapy with or without chemotherapy for nasopharyngeal carcinoma: radiation therapy oncology group phase II trial 0225 . J Clin Oncol. 2009; 27:3684-3690.

3. Kam MK, Chau RM, Suen J, Choi PH and Teo PM. Intensity-modulated radiotherapy in nasopharyngeal carcinoma: dosimetric advantage over conventional plans and feasibility of dose escalation. Int J Radiat Oncol Biol Phys. 2003; 56:145-157.

4. Lu SH, Cheng JC, Kuo SH, Lee JJ, Chen LH, Wu JK, Chen YH, Chen WY, Wen SY, Chong FC, Wu CJ and Wang $\mathrm{CW}$. Volumetric modulated arc therapy for nasopharyngeal carcinoma: A dosimetric comparison with TomoTherapy and step-and-shoot IMRT. Radiother Oncol. 2012; 104:323330 .

5. Chen JL, Huang YS, Kuo SH, Chen YF, Hong RL, Ko JY, Lou PJ, Tsai CL, Chen WY and Wang CW. Intensitymodulated radiation therapy for $\mathrm{T} 4$ nasopharyngeal carcinoma. Treatment results and locoregional recurrence. Strahlenther Onkol. 2013; 189:1001-1008.

6. Lin L, Yao JJ, Zhou GQ, Guo R, Zhang F, Zhang Y, Xu L, Zhang LL, Lin AH, Ma J and Sun Y. The efficacy and toxicity of individualized intensitymodulated radiotherapy based on the tumor extension patterns of nasopharyngeal carcinoma. Oncotarget. 2016; 7: 20680-90. doi: 10.18632/ oncotarget.8004.

7. Fang FM, Chien CY, Tsai WL, Chen HC, Hsu HC, Lui CC, Huang TL and Huang HY. Quality of life and survival outcome for patients with nasopharyngeal carcinoma receiving three-dimensional conformal radiotherapy vs. intensity-modulated radiotherapy-a longitudinal study. Int J Radiat Oncol Biol Phys. 2008; 72:356-364.

8. Kuang WL, Zhou Q and Shen LF. Outcomes and prognostic factors of conformal radiotherapy versus intensitymodulated radiotherapy for nasopharyngeal carcinoma. Clinical \& translational oncology. 2012; 14:783-790.

9. Lai SZ, Li WF, Chen L, Luo W, Chen YY, Liu LZ, Sun Y, Lin AH, Liu MZ and Ma J. How does intensitymodulated radiotherapy versus conventional twodimensional radiotherapy influence the treatment results in nasopharyngeal carcinoma patients? Int J Radiat Oncol Biol Phys. 2011; 80:661-668.

10. Peng G, Wang T, Yang KY, Zhang S, Zhang T, Li Q, Han $\mathrm{J}$ and $\mathrm{Wu} \mathrm{G}$. A prospective, randomized study comparing outcomes and toxicities of intensity-modulated radiotherapy $v s$. conventional two-dimensional radiotherapy for the treatment of nasopharyngeal carcinoma. Radiother Oncol. 2012; 104:286-293.
11. Zhang B, Mo Z, Du W, Wang Y, Liu L and Wei Y. Intensity-modulated radiation therapy versus $2 \mathrm{D}-\mathrm{RT}$ or 3D-CRT for the treatment of nasopharyngeal carcinoma: A systematic review and meta-analysis. Oral oncology. 2015; 51:1041-1046.

12. OuYang PY, Shi D, Sun R, Zhu YJ, Xiao Y, Zhang LN, Zhang XH, Chen ZY, Lan XW, Tang J, Gao YH, Ma J, Deng $\mathrm{W}$ and Xie FY. Effect of intensity-modulated radiotherapy versus two-dimensional conventional radiotherapy alone in nasopharyngeal carcinoma. Oncotarget. 2016; 7:33408-17. doi: 10.18632/oncotarget.8573.

13. Zhang MX, Li J, Shen GP, Zou X, Xu JJ, Jiang R, You R, Hua YJ, Sun Y, Ma J, Hong MH and Chen MY. Intensity-modulated radiotherapy prolongs the survival of patients with nasopharyngeal carcinoma compared with conventional two-dimensional radiotherapy: A 10-year experience with a large cohort and long follow-up. Eur J Cancer. 2015; 51:2587-2595.

14. Zhou GQ, Yu XL, Chen M, Guo R, Lei Y, Sun Y, Mao YP, Liu LZ, Li L, Lin AH and Ma J. Radiation-induced temporal lobe injury for nasopharyngeal carcinoma: a comparison of intensity-modulated radiotherapy and conventional twodimensional radiotherapy. PloS one. 2013; 8:e67488.

15. Peng H, Chen L, Zhang Y, Guo R, Li WF, Mao YP, Tan LL, Sun Y, Zhang F, Liu LZ, Tian L, Lin AH and Ma J. Survival analysis of patients with advanced-stage nasopharyngeal carcinoma according to the Epstein-Barr virus status. Oncotarget. 2016; 7:24208-16. doi: 10.18632/ oncotarget.8144.

16. Wang WY, Lin TY, Twu CW, Tsou HH, Lin PJ, Liu YC, Huang JW, Hsieh HY and Lin JC. Long-term clinical outcome in nasopharyngeal carcinoma patients with post-radiation persistently detectable plasma EBV DNA. Oncotarget. 2016; 7:42608-42616. doi: 10.18632/ oncotarget.9323.

17. Zhang Y, Li WF, Mao YP, Guo R, Tang LL, Peng H, Sun Y, Liu Q, Chen L and Ma J. Risk stratification based on change in plasma Epstein-Barr virus DNA load after treatment in nasopharyngeal carcinoma. Oncotarget. 2016; 7:9576-9585. doi: 10.18632/oncotarget.7083.

18. Chen L, Mao YP, Xie FY, Liu LZ, Sun Y, Tian L, Tang LL, Lin AH, Li L and Ma J. The seventh edition of the UICC/AJCC staging system for nasopharyngeal carcinoma is prognostically useful for patients treated with intensitymodulated radiotherapy from an endemic area in China. Radiother Oncol. 2012; 104:331-337.

19. Cox JD, Stetz J and Pajak TF. Toxicity criteria of the Radiation Therapy Oncology Group (RTOG) and the European Organization for Research and Treatment of Cancer (EORTC). Int J Radiat Oncol Biol Phys. 1995; 31:1341-1346. 